\title{
Centrality, transverse momentum and collision energy dependence of the Tsallis parameters in relativistic heavy-ion collisions
}

\author{
Rajendra Nath Patra ${ }^{1,2,3, a} \mathbb{B}_{D}$, Bedangadas Mohanty ${ }^{4}$, Tapan K. Nayak ${ }^{2,4}$ \\ ${ }^{1}$ Bogolyubov Institute for Theoretical Physics, National Academy of Sciences of Ukraine, Kiev, Ukraine \\ 2 CERN , CH 1211, Geneva, Switzerland \\ 3 University of Jammu, Jammu 180006, India \\ 4 National Institute of Science Education and Research, Jatni 752050, India
}

Received: 5 August 2020 / Accepted: 9 June 2021

(C) The Author(s) 2021

\begin{abstract}
The thermodynamic properties of matter created in high-energy heavy-ion collisions have been studied in the framework of the non-extensive Tsallis statistics. The transverse momentum $\left(p_{\mathrm{T}}\right)$ spectra of identified charged particles (pions, kaons, protons) and all charged particles from the available experimental data of $\mathrm{Au}-\mathrm{Au}$ collisions at the Relativistic Heavy Ion Collider (RHIC) energies and $\mathrm{Pb}-\mathrm{Pb}$ collisions at the Large Hadron Collider (LHC) energies are fitted by the Tsallis distribution. The fit parameters, $q$ and $T$, measure the degree of deviation from an equilibrium state and the effective temperature of the thermalized system, respectively. The $p_{\mathrm{T}}$ spectra are well described by the Tsallis distribution function from peripheral to central collisions for the wide range of collision energies, from $\sqrt{s_{\mathrm{NN}}}=7.7$ $\mathrm{GeV}$ to $5.02 \mathrm{TeV}$. The extracted Tsallis parameters are found to be dependent on the particle species, collision energy, centrality, and fitting ranges in $p_{\mathrm{T}}$. For central collisions, both $q$ and $T$ depend strongly on the fit ranges in $p_{\mathrm{T}}$. For most of the collision energies, $q$ remains almost constant as a function of centrality, whereas $T$ increases from peripheral to central collisions. For a given centrality, $q$ systematically increases as a function of collision energy, whereas $T$ has a decreasing trend. A profile plot of $q$ and $T$ with respect to collision energy and centrality shows an anti-correlation between the two parameters.
\end{abstract}

\section{Introduction}

Relativistic heavy-ion collisions produce matter at extreme conditions of temperatures and energy densities in the form of quark-gluon plasma (QGP). The QGP state is formed at the early stages of the collision, which survives for a very short span of time ( $7-10 \mathrm{fm} / c)$, after which the matter gets transformed rapidly to a system of hadron gas. The information about the initial condition of the system gets mostly lost by multi-partonic interactions throughout the evolution of the collision. The final state behavior of such a colliding system can be obtained from the measurement of the number and identity of produced particles, along with their energy and momentum spectra. The final state information is nevertheless most useful

\footnotetext{
a e-mails: rajendra.nath.patra@cern.ch ; rajendrapatra07@gmail.com (corresponding author)
} 
for understanding the particle production mechanisms and the nature of the matter produced in these high-energy collisions.

The space-time evolution of the hot and dense system produced in the collision ceases at two symbolic freeze-out conditions, namely the chemical freeze-out and kinetic freeze-out. The colliding medium first reaches chemical equilibrium and then cools down by expansion. Due to this expansion of the system, the inelastic collisions cease when the mean free path for the interactions becomes comparable to the system size. This is known as the chemical freeze-out, at which point the abundances of different particle species become constant. After this stage, even if the relative fractions of the particles are constant, these particles continue to interact till a point where the final state interactions between the hadrons are no longer effective. After this kinetic freeze-out, all the interactions cease and the (transverse) momentum spectra of the particles remain unchanged. Therefore, transverse momentum $\left(p_{\mathrm{T}}\right)$ spectra of produced particles constitute some of the basic measurements to extract the kinetic freeze-out condition of the systems produced in high-energy collisions.

Several different standard statistical model fits using Boltzmann-Gibbs (BG), FermiDirac, Bose-Einstein distributions, $m_{\mathrm{T}}$-exponential distribution [1,2] Erlang distribution [3], Tsallis distribution [4-8], and other kinds of distribution functions have been used to describe the $p_{\mathrm{T}}$ spectra and extract physical parameters. The BG statistical model has attained a huge success in explaining the thermodynamic properties in different physical systems. However, the BG thermal (exponential) model is inappropriate at high transverse momenta in highenergy collision systems as it cannot explain the high transverse momentum range which is mostly followed by the inverse power-law behavior [9-11]. A distribution following the nonextensive Tsallis statistics $[12,13]$ has been shown to provide a better description of the nature of the non-exponential transverse momentum spectrum. The description of particle spectra using the Tsallis distribution has gained lots of interest recently as it can describe the spectra at high $p_{\mathrm{T}}$. The Tsallis distribution is found to be very successful in describing the spectra at high $p_{\mathrm{T}}$ ranges in proton-proton $(p p)$ collisions $[4,5]$. The $p_{\mathrm{T}}$ spectra of identified particles in $p p$ system at different collision energies can be explained by the Tsallis distribution function as presented in Ref. [6,14], whereas the multiplicity dependence of all charged particles spectra with the Tsallis framework is reported in Ref. [15].

In case of heavy-ion collisions, the transverse momentum spectra have traditionally been fitted by blast-wave model [16] [1,2,17-19]. This model has a simple assumption that there is a kinetic freeze-out of the colliding medium at temperature $T_{\text {kin }}$ and particles are moving with a common collective radial flow velocity $(\beta)$. However, the blast-wave model cannot be used to describe the spectra at mid- to high- $p_{\mathrm{T}}$ ranges. Recent studies [7] have found that the Tsallis statistics also works in heavy-ion collisions providing good fitting of the $p_{\mathrm{T}}$ spectra of all charged particles. Since these fits do not account for the radial flow, the temperatures obtained from the Tsallis fits are the effective temperatures, different from the kinetic freezeout temperatures. This effective temperature depends on the particle mass and the collective flow velocity. The intercept in $T=T_{\text {kin }}+1 / 2 m \beta_{\mathrm{T}}^{2}$ is an alternative method [20-22] to find freeze-out temperature where $m$ is the rest mass and $\beta_{\mathrm{T}}$ is the average transverse radial flow velocity.

In the present work, we use the thermodynamically consistent form of the Tsallis distribution [8] to fit the transverse momentum $\left(p_{\mathrm{T}}\right)$ spectra of the identified charged particles with a focus on pions as well as all charged particles in heavy-ion collision systems corresponding to eight different collision energies at RHIC and two collision energies at the LHC. For each of the spectrum, we obtain the Tsallis fit parameters, $q$, which expresses the degree of deviation from an equilibrium state, and $T$ as the effective temperature. The dependencies of the fitting parameters on collision centrality, collision energy and fitting ranges in $p_{\mathrm{T}}$ are 
investigated and will be presented. The relative success of Tsallis distribution for different particles in heavy-ion system will be also discussed. In Sect. 2, we discuss the formulation of the Tsallis distribution and the methodology of fitting the $p_{\mathrm{T}}$ spectra. In Sect. 3, we present Tsallis fittings of the $p_{\mathrm{T}}$ spectra at different centrality, energy and $p_{\mathrm{T}}$ ranges. In Sect. 4 , we present the Tsallis fit parameters and discuss their relevances for different collision energies. A discussion on the results is given in Sect. 5. Finally, the paper is summarized in Sect. 6.

\section{Tsallis statistics and methodology}

The non-extensive Tsallis form of the Boltzmann-Gibbs distribution in terms of energy $(E)$, effective temperature $(T)$, and chemical potential $(\mu)$ of a system can be expressed as $[12,13]$,

$$
f(E, q, T, \mu) \equiv \exp _{\mathrm{q}}\left(-\frac{E-\mu}{T}\right) \equiv\left(1+(q-1) \frac{E-\mu}{T}\right)^{-\frac{1}{q-1}} .
$$

The $\exp _{\mathrm{q}}(x)$ has the form,

$$
\exp _{\mathrm{q}}(x) \equiv \begin{cases}(1+(q-1) x)^{\frac{1}{q-1}} & \text { if } x>0 \\ (1+(1-q) x)^{\frac{1}{1-q}} & \text { if } x \leq 0\end{cases}
$$

where $q$ is the entropy index which measures the degree of non-additivity of the entropy or deviation from the equilibrium of the system. In general, $q \geq 1$. In equilibrium case, i.e., in the limit $q \rightarrow 1, \exp _{\mathrm{q}}(x) \rightarrow \exp (x)$. Therefore, Eq. 1 is simplified to the extensive Boltzmann-Gibbs form,

$$
f_{q \rightarrow 1}(E, q, T \mu) \equiv f_{\mathrm{BG}}(E, T, \mu) \equiv \exp \left(-\frac{E-\mu}{T}\right) .
$$

The Tsallis form with thermodynamical consideration of the invariant momentum distribution of the particles has been introduced and discussed in $[8,23]$. The particle number density $(n)$ can be expressed using Tsallis statistics as,

$$
n=g \int \frac{d^{3} p}{(2 \pi)^{3}} f^{q},
$$

where $g$ is the degeneracy factor of the particles. The expression of the invariant momentum distribution can be obtained from the particle number density as given in Eq. 4 as,

$$
E \frac{d^{3} N}{d p^{3}}=\frac{g V}{(2 \pi)^{3}} E\left(1+(q-1) \frac{E-\mu}{T}\right)^{-\frac{q}{q-1}} .
$$

Here $N$ is the particle number, and $V$ is refereed as the volume of the system which depends on the $q$. Therefore, $V$ is not necessarily the volume of the system, but serves as a normalization factor of the Tsallis distribution. For non-vanishing chemical potential, Eq. 5 can be rewritten in terms of transverse mass $\left(m_{\mathrm{T}}\right)$ and rapidity $(y)$ in the form,

$$
\begin{gathered}
\frac{1}{2 \pi p_{\mathrm{T}}} \frac{d^{2} N}{d p_{\mathrm{T}} d y}=\frac{g V}{(2 \pi)^{3}} m_{\mathrm{T}} \cosh (y) \\
\left(1+(q-1) \frac{m_{\mathrm{T}} \cosh (y)-\mu}{T}\right)^{-\frac{q}{q-1}} .
\end{gathered}
$$


The invariant yield, $\frac{1}{2 \pi p_{\mathrm{T}}} \frac{d^{2} N}{d p_{\mathrm{T}} d y}$, is an experimentally measured observable. Therefore, the Tsallis parameters $q, T$ can be obtained from experimentally measured invariant momentum distributions using the fitting function as given in Eq. 6, with $\mu$ as an input.

A different type of Tsallis distribution is used by the STAR [24], PHENIX [25, 26], ATLAS [27], CMS [28] and ALICE [29-32] collaborations as in the expression below,

$$
\frac{1}{2 \pi p_{\mathrm{T}}} \frac{d^{2} N}{d p_{\mathrm{T}} d y}=\frac{1}{2 \pi} \frac{d N}{d y} \frac{(n-1)(n-2)}{n C(n C+m(n-2))}\left(1+\frac{m_{\mathrm{T}}-m}{n C}\right)^{-n},
$$

where $n, C$ are the fitting parameters and $m$ is the rest mass of the particle. At mid-rapidity and zero chemical potential, Eq. 7 has same dependency on transverse momentum as in Eq. 6 except the additional $m_{\mathrm{T}}$ dependency. The incorporation of $m_{\mathrm{T}}$ in Eq. 6 has more consistent behavior of $q$ and $T$, whereas no clear pattern of $n$ and $C$ can be found [8]. In this present study, Tsallis distribution of thermodynamical consistent form given in Eq. 6 has been used. The conversion from rapidity to pseudo-rapidity phase-space is made using a Jacobian $(J(y, \eta))$ of the form,

$$
\frac{d N}{d p_{\mathrm{T}} d \eta}=\sqrt{1-\frac{m^{2}}{m_{\mathrm{T}}^{2} \cosh ^{2}(y)}} \frac{d N}{d y d p_{\mathrm{T}}} .
$$

At mid-rapidity ( $y \approx 0$ ), this can be simplified as

$$
\frac{d N}{d p_{\mathrm{T}} d \eta}=\frac{p_{\mathrm{T}}}{m_{\mathrm{T}}} \frac{d N}{d y d p_{\mathrm{T}}} .
$$

The expression of the Tsallis distribution for all charged particle pseudo-rapidity distribution at mid-rapidity can be given as,

$$
\frac{d^{2} N_{\mathrm{ch}}}{d p_{\mathrm{T}} d \eta}=2 p_{\mathrm{T}}^{2} \frac{V}{(2 \pi)^{2}} \sum_{i=1}^{3} g_{i}\left(1+(q-1) \frac{m_{\mathrm{T}, \mathrm{i}}-\mu}{T}\right)^{-\frac{q}{q-1}} .
$$

Here, the sum runs for three kind of the particles $\pi^{+}, K^{+}$, and $p$ considering that those are the most abundant in productions. The factor 2 in front of the right side takes into account of the associated anti-particles, $\pi^{-}, K^{-}$, and $\bar{p}$. The degeneracy factors $(g)$ are unity for pions and kaons and 2 for protons. At mid-rapidity, Eq. 6 reduces to the form,

$$
\frac{1}{2 \pi p_{\mathrm{T}}} \frac{d^{2} N}{d p_{\mathrm{T}} d y}=\frac{g V}{(2 \pi)^{3}} m_{\mathrm{T}}\left(1+(q-1) \frac{m_{\mathrm{T}}-\mu}{T}\right)^{-\frac{q}{q-1}} .
$$

This equation (11) has been used to fit invariant momentum spectra of identified particles. The centrality dependent finite chemical potential $\mu$ has been considered for the RHIC energy $[2,33]$, whereas at LHC energies $\mu$ has been approximated to zero.

\section{Fits of the $p_{\mathrm{T}}$ spectra}

Detailed analysis of the transverse momentum spectra of produced particles provides important information regarding the particle production mechanisms. We have fitted the $p_{\mathrm{T}}$ spectra of identified and all charged particles in heavy-ion collisions at RHIC and LHC. At RHIC, the experimental data of $\mathrm{Au}-\mathrm{Au}$ collisions from the beam energy scan (BES-I) program are available for $\sqrt{s_{N N}}=7.7,11.5,14.519 .6,27,39 \mathrm{GeV}[2,17]$ as well as at 62.4 and at the top energy of $200 \mathrm{GeV}[34,35]$. Fine centrality binning data from PHENIX collaboration at 
$\sqrt{s_{N N}}=200 \mathrm{GeV}$ [20] are also fitted. At the LHC, data published by the ALICE collaboration for $p_{\mathrm{T}}$ spectra of identified charged particles in $\mathrm{Pb}-\mathrm{Pb}$ collisions at $\sqrt{s_{N N}}=2.76 \mathrm{TeV}$ $[18,36]$ and at $5.02 \mathrm{TeV}$ [19] have been included in our study. In addition, $p_{\mathrm{T}}$ spectra of all charged particles $\left(N_{\mathrm{ch}}\right)$ measured by ALICE at $\sqrt{s_{N N}}=2.76$ and $5.02 \mathrm{TeV}[37,38]$ are also used. In Table 1, we summarize the experimental datasets used in the present study in terms of collision energy, colliding system, pseudo-rapidity $(\eta)$ range, $p_{\mathrm{T}}$ range, and centrality binning in percentage of the cross section. For the experimental data, the systematic and statistical uncertainties are added in quadrature.

The fits to the transverse momentum spectra at all collision energies as given in Table 1 are performed by the expression of Tsallis distribution as in Eq. 11. The transverse momentum spectra with Tsallis fitting of positively charged pions $\left(\pi^{+}\right)$at different centrality bins in Au-Au collisions at $\sqrt{s_{N N}}=7.7 \mathrm{GeV}$ to $39 \mathrm{GeV}$ measured by the STAR collaboration from the RHIC BES-I $[2,17]$ program are shown in Fig. 1. The data are available for $p_{\mathrm{T}}$ range up to $2 \mathrm{GeV} / c$, and so the fits, represented by the solid lines, are also made up to this $p_{\mathrm{T}}$ range. The Tsallis distribution provides very good fits of the data at all centralities, which is evident from the ratio of the experimental data to the fit value as shown in the lower panel of the

Table 1 Collision energy, collision species, observed particles, centrality binning, pseudo-rapidity, and transverse momentum range of $p_{\mathrm{T}}$ spectra from the STAR, PHENIX and ALICE experiments

\begin{tabular}{|c|c|c|c|c|c|}
\hline$\sqrt{s_{N N}}(\mathrm{GeV})$ & $\begin{array}{l}\text { Collision } \\
\text { system }\end{array}$ & Particles & $|y|(|\eta|)$ & Centrality (\%) & Experiment \\
\hline $\begin{array}{l}7.7,11.5,19.6 \\
27,39\end{array}$ & $\mathrm{Au}-\mathrm{Au}$ & $\begin{array}{l}\pi^{+}, \pi^{-}, \mathrm{K}^{+}, \mathrm{K}^{-} \\
\quad \mathrm{p}, \bar{p}\end{array}$ & 0.1 & $\begin{array}{l}0-5,5-10,10-20,20-30 \\
\quad 30-40,40-50,50-60,60-70 \\
70-80\end{array}$ & STAR [2] \\
\hline 14.5 & $\mathrm{Au}-\mathrm{Au}$ & $\begin{array}{l}\pi^{+}, \pi^{-}, \mathrm{K}^{+}, \mathrm{K}^{-} \\
\quad \mathrm{p}, \bar{p}\end{array}$ & 0.1 & $\begin{array}{l}0-5,5-10,10-20,20-30 \\
\quad 30-40,40-50,50-60,60-70 \\
70-80\end{array}$ & STAR [17] \\
\hline 62.4 & $\mathrm{Au}-\mathrm{Au}$ & $\pi^{+}, \pi^{-}, \mathrm{p}, \bar{p}$ & 0.5 & $0-10,10-20,20-40,40-80$ & STAR [34] \\
\hline 200 & $\mathrm{Au}-\mathrm{Au}$ & $\pi^{+}, \pi^{-}, \mathrm{p}, \bar{p}$ & 0.5 & $\begin{array}{l}0-12,10-20,20-40,40-60 \\
\quad 60-80\end{array}$ & STAR [35] \\
\hline 200 & $\mathrm{Au}-\mathrm{Au}$ & $\begin{array}{l}\pi^{+}, \pi^{-}, \mathrm{K}^{+}, \mathrm{K}^{-} \\
\quad \mathrm{p}, \bar{p}\end{array}$ & 0.26 & $\begin{array}{l}0-5,5-10,10-15,15-20 \\
\quad 20-30,30-40,40-50,50-60 \\
60-70,70-80,80-92\end{array}$ & PHENIX [20] \\
\hline 2760 & $\mathrm{~Pb}-\mathrm{Pb}$ & $\begin{array}{l}\pi^{+}, \pi^{-}, \mathrm{K}^{+}, \mathrm{K}^{-} \\
\quad \mathrm{p}, \bar{p}\end{array}$ & 0.5 & $\begin{array}{l}0-5,5-10,10-20,20-30 \\
\quad 30-40,40-50,50-60,60-70 \\
70-80,80-90\end{array}$ & ALICE [18] \\
\hline 2760 & $\mathrm{~Pb}-\mathrm{Pb}$ & $\begin{array}{l}\pi^{+}+\pi^{-} \\
\mathrm{K}^{+}+\mathrm{K}^{-}, \mathrm{p}+\bar{p}\end{array}$ & 0.5 & $\begin{array}{l}0-5,5-10,10-20,20-30 \\
\quad 30-40,20-40,40-50,40-60 \\
60-80\end{array}$ & ALICE [36] \\
\hline 2760 & $\mathrm{~Pb}-\mathrm{Pb}$ & $\mathrm{N}_{\mathrm{ch}}$ & 0.8 & $\begin{array}{l}0-5,5-10,10-20,20-30 \\
\quad 30-40,40-50,50-60,60-70 \\
70-80\end{array}$ & ALICE [37] \\
\hline 5020 & $\mathrm{~Pb}-\mathrm{Pb}$ & $\begin{array}{l}\pi^{+}+\pi^{-} \\
\mathrm{K}^{+}+\mathrm{K}^{-}, \mathrm{p}+\bar{p}\end{array}$ & 0.5 & $\begin{array}{l}0-5,5-10,10-20,20-30 \\
30-40,40-50,50-60,60-70 \\
70-80,80-90\end{array}$ & ALICE [19] \\
\hline 5020 & $\mathrm{~Pb}-\mathrm{Pb}$ & $\mathrm{N}_{\mathrm{ch}}$ & 0.8 & $\begin{array}{l}0-5,5-10,10-20,20-30 \\
\quad 30-40,40-50,50-60,60-70 \\
70-80\end{array}$ & ALICE [38] \\
\hline
\end{tabular}



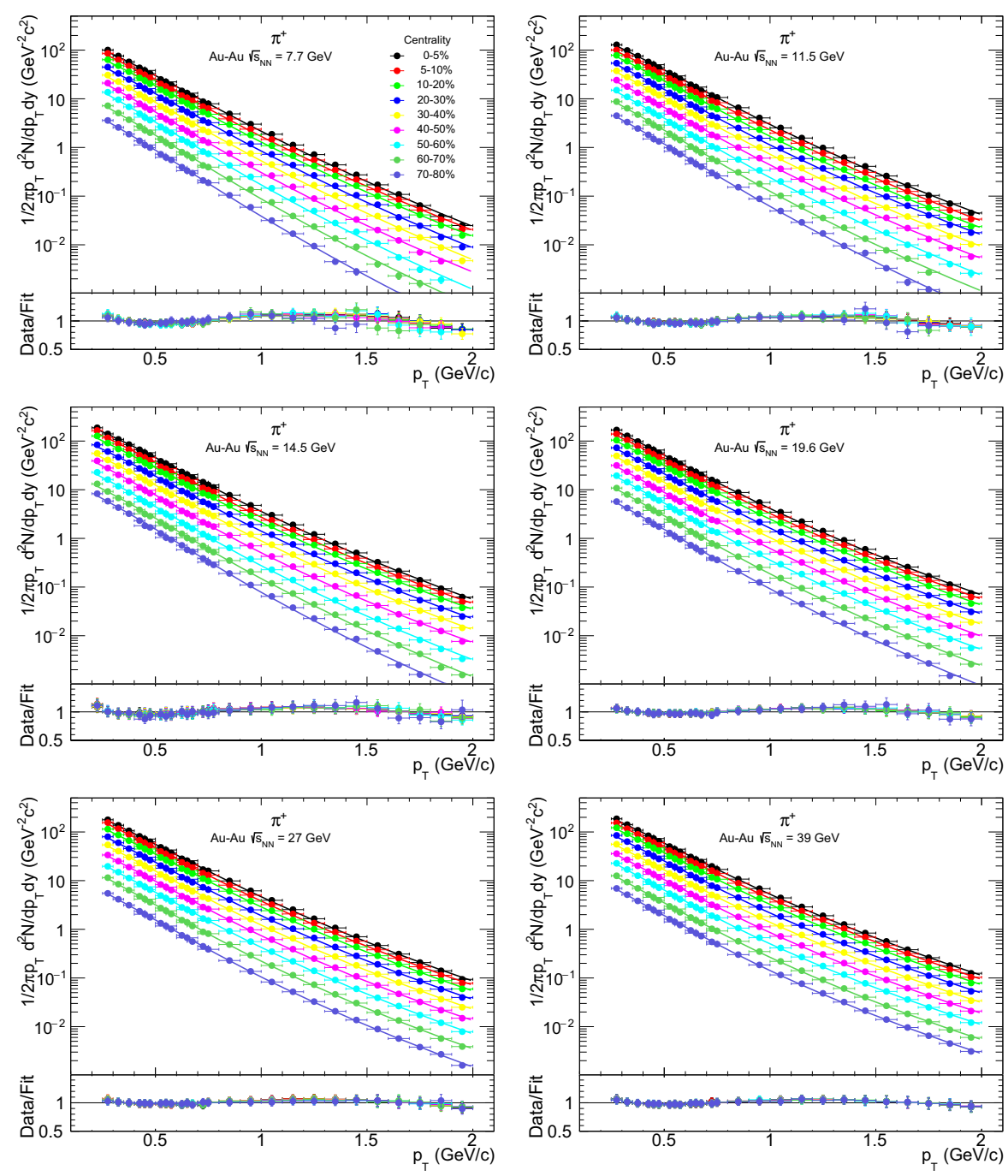

Fig. 1 Transverse momentum distributions of $\pi^{+}$for different centralities at $\sqrt{s_{N N}}=7.7,11.5,14.5$, 19.6, 27, $39 \mathrm{GeV}$, measured by the STAR experiment [2,17]. The solid lines represent fitting by the Tsallis distribution as given in Eq. 11. The lower parts of the figures represent the ratios of the data to that of the fitting values

figure. The maximum deviations of the ratios from unity are within $\sim 10-15 \%$. The fits of the $p_{\mathrm{T}}$ spectra of $\pi^{-}$distributions are of similar quality to the $\pi^{+}$distributions.

Figure 2 shows the $p_{\mathrm{T}}$ spectra along with the corresponding Tsallis fits for Au-Au collisions at $\sqrt{s_{N N}}=62.4$ and $200 \mathrm{GeV}$ from STAR [34,35] experiment with the available $p_{\mathrm{T}}$ ranges up to $10 \mathrm{GeV} / c$ and $12 \mathrm{GeV} / c$, respectively. The left panels of the figure show the fits of the $p_{\mathrm{T}}$ range up to $2 \mathrm{GeV} / c$, and the right panels provide the Tsallis fitting of the entire $p_{\mathrm{T}}$ spectrum. The ratios of the data to fit values are shown in the lower part of the figures. We observe that the quality of the Tsallis distribution fits shown in the left panels are quite good (with maximum deviations within $\sim 10-15 \%$ ) for fits up to low- $p_{\mathrm{T}}$ region of $2 \mathrm{GeV} / \mathrm{c}$. 

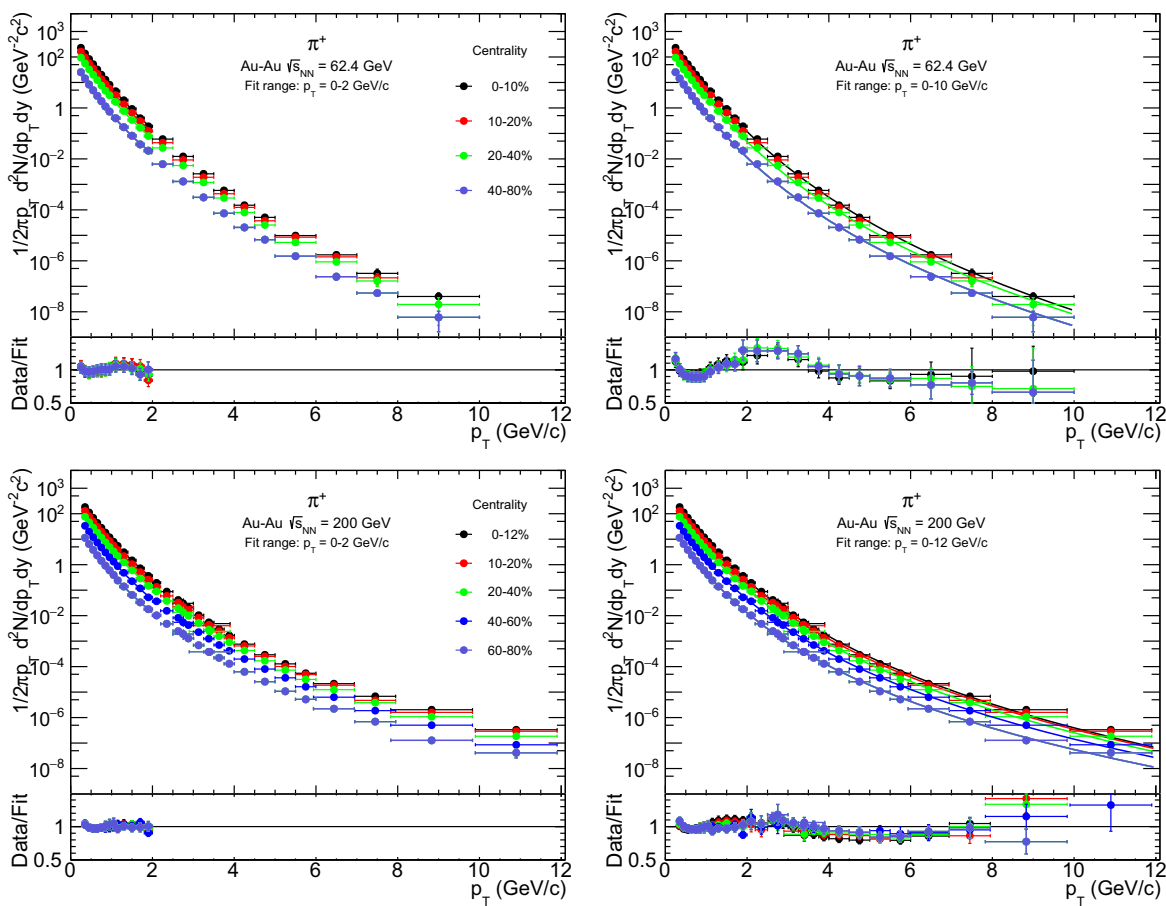

Fig. 2 Transverse momentum distributions of $\pi^{+}$for different centralities at $\sqrt{s_{N N}}=62.4$ and $200 \mathrm{GeV}$ measured by the STAR experiment $[34,35]$. The left and right panels correspond to fitting ranges of $p_{\mathrm{T}}$ up to $2 \mathrm{GeV} / c$ and up to the full $p_{\mathrm{T}}$ range, respectively. The solid lines represent fitting by the Tsallis distribution as given in Eq. 11. The lower parts of the figures represent the the ratios of the data to that of the fitting values
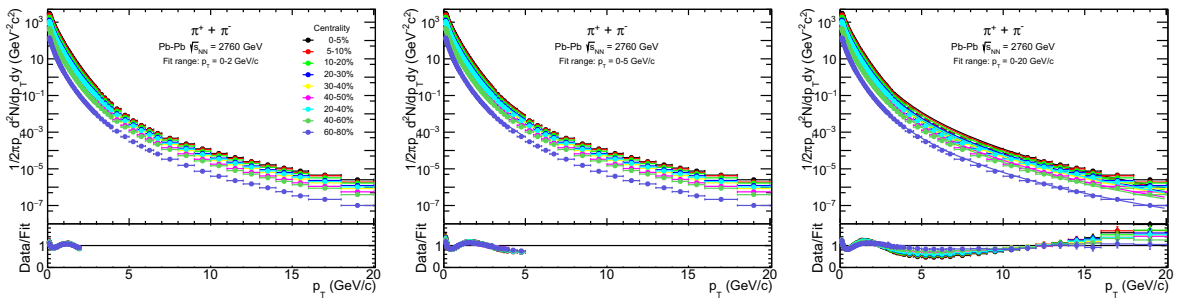

Fig. 3 Transverse momentum distribution of $\pi^{+}+\pi^{-}$for different centralities at $\sqrt{s_{N N}}=2.76 \mathrm{TeV}$ measured by the ALICE experiment [36]. The left, middle, and right panels correspond to fitting ranges of $p_{\mathrm{T}}$ up to $2 \mathrm{GeV} / c, 5 \mathrm{GeV} / c$, and $20 \mathrm{GeV} / c$, respectively. The solid lines represent fitting by the Tsallis distribution as given in Eq. 11. The lower parts of the figures represent the ratios of the data to that of the fitting values

Fits to higher $p_{\mathrm{T}}$ as shown in the right panels are not as good (the maximum deviations are larger, up to about $30 \%$ ). The fit parameters turn out to be dependent on the fit ranges in $p_{\mathrm{T}}$. The results of the fits are discussed in detail in the next section.

In Fig. 3, we present the transverse momentum spectra of all charged pions $\left(\pi^{+}+\pi^{-}\right)$ for different centrality bins for Pb-Pb collisions at $\sqrt{s_{N N}}=2.76 \mathrm{TeV}$ from the ALICE [36] collaboration. The fits with the Tsallis distribution function have been shown for three different $p_{\mathrm{T}}$ ranges, where the left, middle, and the right plots in the figure correspond to 


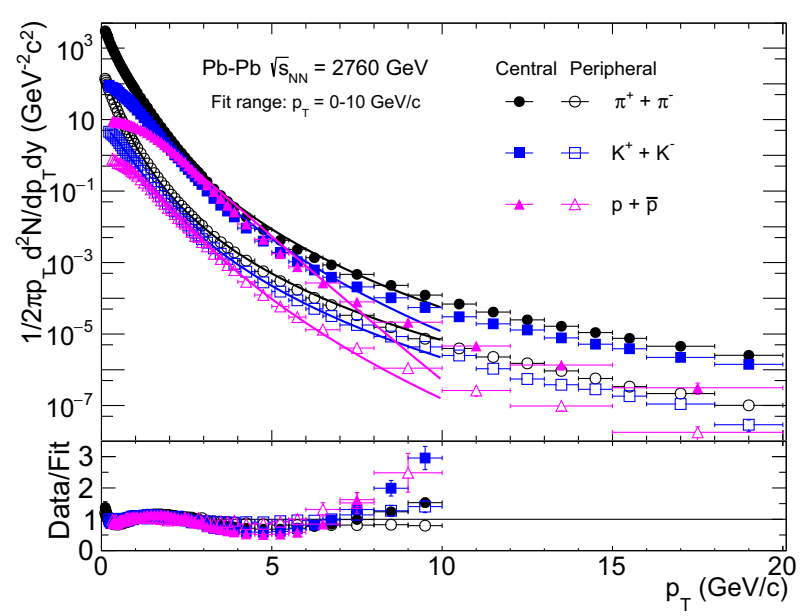

Fig. 4 Transverse momentum distribution of $\pi^{+}+\pi^{-}, \mathrm{K}^{+}+\mathrm{K}^{-}$and $\mathrm{p}+\mathbb{\mathrm { P }}$ for most central $(0-5 \%$, solid markers) and peripheral (70-80\%, open markers) collisions at $\sqrt{s_{N N}}=2.76 \mathrm{TeV}$ measured by the ALICE experiment [36]. The solid lines represent the fits up to $p_{\mathrm{T}}$ of $10 \mathrm{GeV} / c$ using the Tsallis distribution as given in Eq. 11. The lower panel of the figure represent the ratios of the data to that of the fitting values

$p_{\mathrm{T}}$ ranges up to $2 \mathrm{GeV} / c, 5 \mathrm{GeV} / c$ and $20 \mathrm{GeV} / c$, respectively. The lower parts of the plots give the ratio of the data to the fitted values. These ratios show that the fitting is in good agreement with the data for peripheral events, whereas some deviations have been observed for central collisions and at large $p_{\mathrm{T}}$. Furthermore, it is observed that the fit parameters vary according to the fitting ranges in $p_{\mathrm{T}}$. In addition, we have fitted the low- $p_{\mathrm{T}}$ region of these spectra by fixing the parameter $q=1$ which gives the standard BG distribution, (Eq. 3). We observed that fixing the parameter $q$ to unity does not provide a reasonable fit even at low- $p_{\mathrm{T}}$ region, i.e., transverse momentum spectrum cannot be explained by exponential function only. Different studies present that at low- $p_{\mathrm{T}}$ region the spectra are modified by resonance productions [39], which is not considered here in the Tsallis fitting.

The transverse momentum spectra of identified charged pions, kaons, and protons at different collision energies and centralities are fitted using the Tsallis distribution. The fitting of the $p_{\mathrm{T}}$ spectra of $\mathrm{K}^{+}+\mathrm{K}^{-}$and $\mathrm{p}+\bar{p}$ along with $\pi^{+}+\pi^{-}$for $\mathrm{Pb}-\mathrm{Pb}$ collisions at $\sqrt{s_{N N}}=2.76 \mathrm{TeV}$ [36] are shown in Fig. 4 . The fits of the data are performed for $p_{\mathrm{T}}$ ranges up to $10 \mathrm{GeV} / c$ for the most central $(0-5 \%)$ and peripheral $(70-80 \%)$ collisions as presented by the solid and open markers, respectively. The quality of the fits is presented by the ratio of data to fit values at the lower part of the figure. In general, we observe that the data for peripheral collisions are fitted better compared to those of the central collisions. Moreover, the fits are considerably better for pions, then kaons, and finally for protons. The fits diverge gradually from the data with increasing particle masses and centrality at large $p_{\mathrm{T}}$ ranges.

\section{Results of the Tsallis fit parameters}

In this section, we present the results of the fitting of $p_{\mathrm{T}}$ distributions as a function of collision centrality, collision energy as well as the $p_{\mathrm{T}}$ ranges of the fits. The results are presented for the Tsallis parameters, $q, T$, and the normalization volume, $V$. 


\subsection{Centrality dependence of Tsallis parameters}

In the previous section, we have presented $p_{\mathrm{T}}$ distributions for different collision energies and collision centralities. The collision centralities have been shown in terms of the percentage of the cross sections. For a given centrality window, the centrality can also be expressed in terms of average number of participating nucleons $\left(N_{\text {part }}\right)$. As the centrality bins in the data as shown in Table 1 are different, it is useful to express the centrality in terms of $N_{\text {part }}$. In Fig. 5, we present the variation of $q, T$, and $V$ as a function of $N_{\text {part }}$ for positively charged pions at RHIC energies, and for all charged pions at LHC energies. The parameters obtained from $\pi^{+}$and $\pi-$ spectra of ALICE data [18] are similar to the results for all charged pions. All the fits are made for maximum values of $p_{\mathrm{T}}$ up to $2 \mathrm{GeV} / c$. The following observations are made regarding the centrality and collision energy dependence of these parameters:

- The Tsallis parameter, $q$, systematically increases from low to high collision energies. For each collision energy, the variation of $q$ as a function of $N_{\text {part }}$ has been inferred by fitting the dependence with a linear function. We observe that within the given uncertainly, $q$ is independent centrality at RHIC energies. However, for LHC energies $q$ decreases from peripheral to central collisions.

- The Tsallis temperature, $T$, decreases from low to high collision energies. For all collision energies, the values of $T$ increase from peripheral to central collisions.
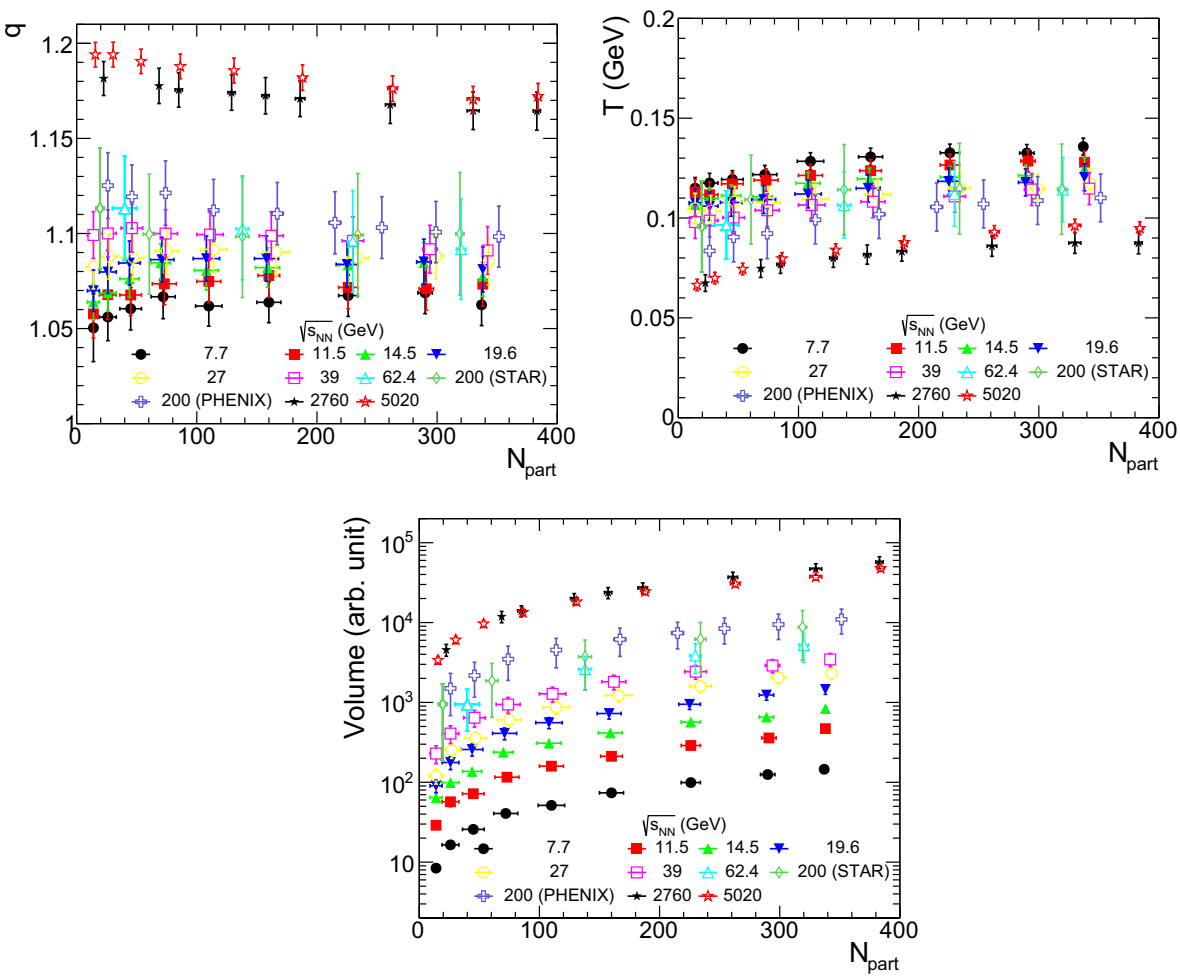

Fig. 5 Tsallis parameters, $q, T$, and the normalization factor, $V$ as a function of number of participants, $N_{\text {part }}$, obtained by fitting the $p_{\mathrm{T}}$ spectra up to $2 \mathrm{GeV} / c$ for $\pi^{+}$at RHIC energies from STAR $[2,17,34,35]$, and PHENIX [20], and all charged pions at LHC energies from ALICE [19,36] 
- The normalization parameter, $V$, increases from low to high collision energies. For all collision energies, $V$ increases monotonically from peripheral to central collisions.

\subsection{Collision energy dependence of Tsallis parameters}

The variations of the Tsallis parameters, $q, T$, and the normalization parameter, $V$, are scanned over the collision energy $\sqrt{s_{N N}}$ from RHIC to LHC energies. The results of the fits to $p_{\mathrm{T}}$ spectra up to a value of $2 \mathrm{GeV} / c$ are presented in Fig. 6 . At RHIC energies, the fits are performed for $\pi^{+}$, and at the LHC energies, the fits are presented for $\pi^{+}+\pi^{-}$. For clarity, results are presented only for central $(0-5 \%, 5-10 \%)$ and peripheral $(70-80 \%)$ collisions. The following observations are made regarding the collision energy dependence of these parameters:

- The parameter $q$ as a function of collision energy shows that for all centralities $q$ increases with the increase in collision energy. A closer look at the centrality dependence shows that at RHIC energy $q$ remains unchanged with the centrality within the uncertainty, whereas, in higher energies, $q$ decreases from peripheral to central collisions.

- The parameter $T$ decreases with the increase in the collision energy. Another important observation is that $T$ increases from peripheral to central collisions at all collision energies.
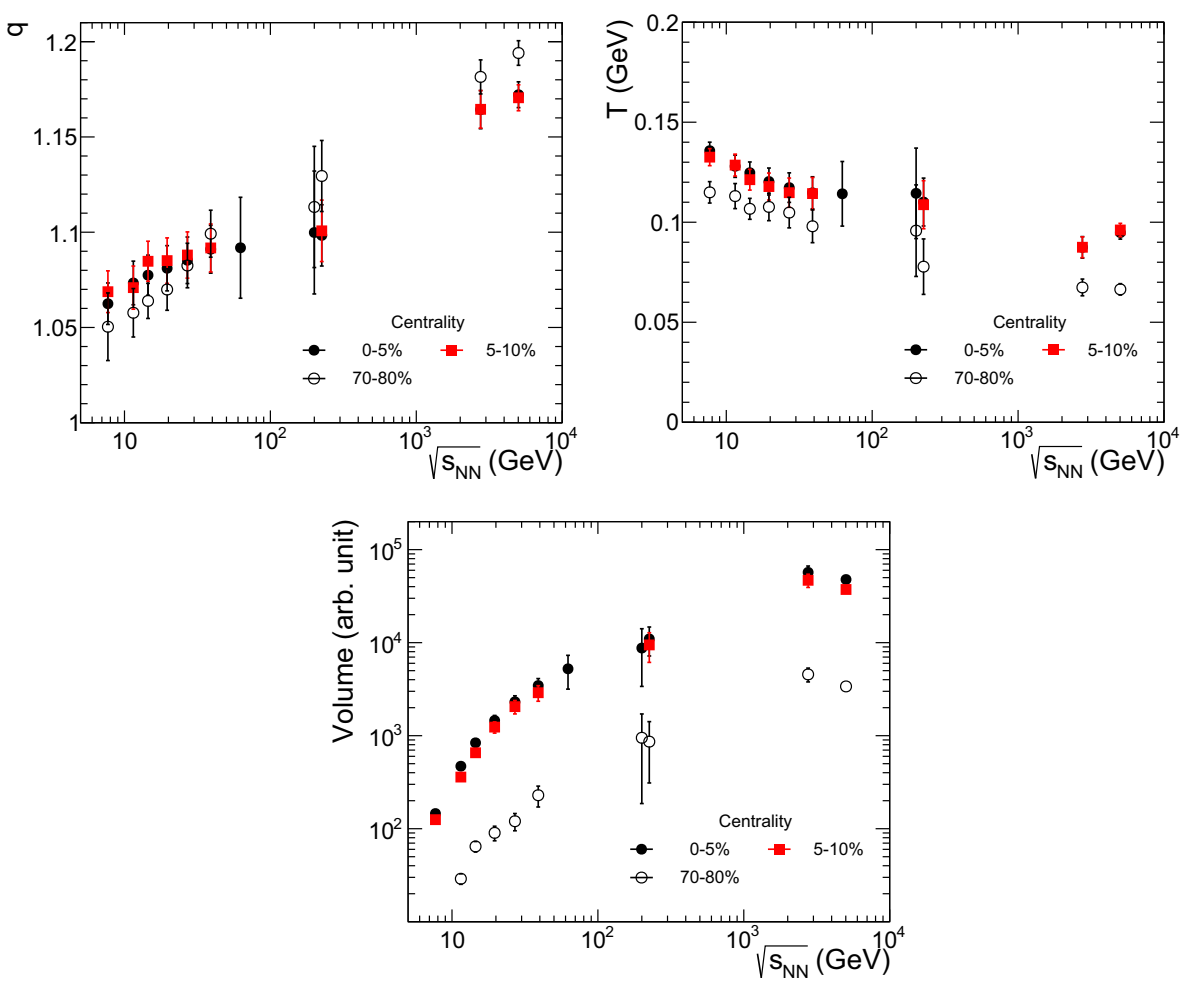

Fig. 6 Tsallis parameters $q, T$, and the normalization factor, $V$ as a function of collision energy, $\sqrt{s_{N N}}$, for three different centralities obtained by fitting the $p_{\mathrm{T}}$ spectra up to $2 \mathrm{GeV} / c$ for $\pi^{+}$at RHIC energies from STAR [2, 17,34,35], and PHENIX [20], and all charged pions at LHC energies from ALICE [19,36]. PHENIX results are shifted to right for clarity 
- The variation of $V$ with collision energy shows that $V$ increases consistently with increasing $\sqrt{s_{N N}}$. This is reasonable as the system size, and hence, particle productions increase with $\sqrt{s_{N N}}$ and centrality.

The usefulness of the Tsallis parameters can be understood by fitting the $p_{\mathrm{T}}$ spectra of kaons and protons along with those of pions. This has been done for RHIC energies from the STAR BES-I [2] and PHENIX [20] datasets and at LHC for the ALICE [19,36] data. The variations of $q$ and $T$ as a function of $\sqrt{s_{N N}}$ are shown in Fig. 7 for all the three identified particles. The parameters presented in the figure correspond to the $p_{\mathrm{T}}$ range up to $2 \mathrm{GeV} / c, 3 \mathrm{GeV} / c$ and $5 \mathrm{GeV} / c$ for pions, kaons, and protons, respectively. The results of the peripheral and central collisions are labeled as open and solid markers, respectively. It has been observed from the figure that for pions and kaons $q$ has an increasing trend with $\sqrt{s_{N N}}$ for both central and peripheral collisions. But for protons, the trend is very different, and at the LHC energies $q$ values are found lower than unity within the fitting range of the proton spectra, which is against the basic assumption of the distribution function. This could be because of the radial flow which has a larger effect on protons that is not considered in the fitting. The temperature $T$ shows a distinct difference for central and peripheral collisions for all the three particle species. For peripheral collisions, $T$ values are similar for all particles. But for central collisions, $T$ shows a strong mass dependency. For pions, $T$ decreases with increase in $\sqrt{s_{N N}}$, whereas $T$ increases for kaons and protons, with unusually high value for protons.

\subsection{Behavior of Tsallis parameters for different $p_{\mathrm{T}}$ ranges}

The Tsallis function has been shown to be successful in fitting $p_{\mathrm{T}}$ distributions over a broad range. In Fig. 8, we present the variation of the Tsallis fit parameters $(q$, and $T)$ for charged pions as a function of centrality for different collision energies and for a set of fitting ranges $(0-2 \mathrm{GeV} / c, 0-5 \mathrm{GeV} / c, 0-7 \mathrm{GeV} / c$, and $0-10 \mathrm{GeV} / c)$. In the figure, different energies are labeled by different markers, whereas $p_{\mathrm{T}}$ ranges are indicated using different color schemes. We observe that:
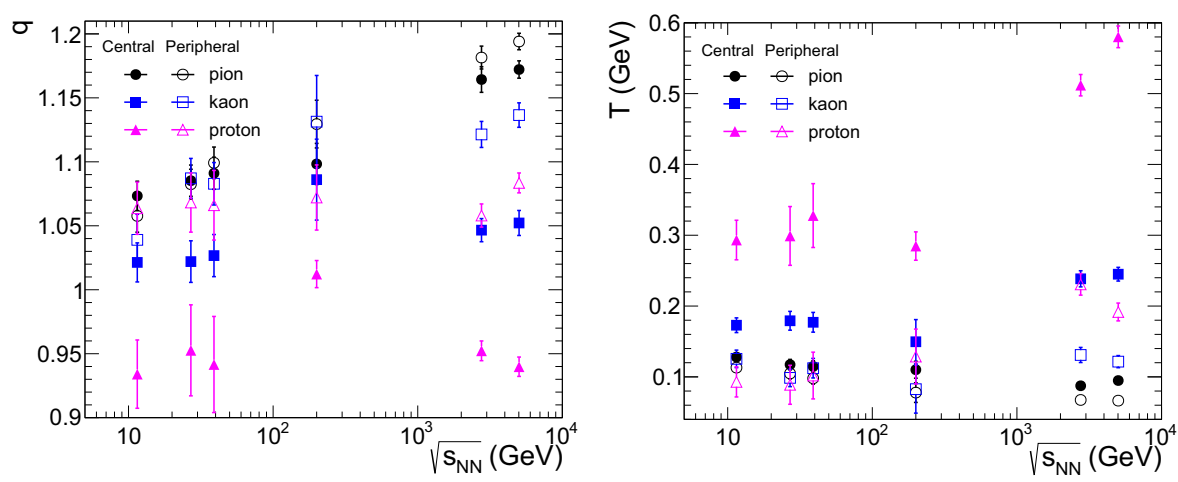

Fig. 7 Tsallis parameters, $q$ and $T$ as a function of collision energy, $\sqrt{s_{N N}}$, for $\pi^{+}\left(\pi^{+}+\right.$ $\left.\pi^{-}\right), \mathrm{K}^{+}\left(\mathrm{K}^{+}+\mathrm{K}^{-}\right)$and $\mathrm{p}(\mathrm{p}+\overline{\mathrm{p}})$ for fits up to $p_{\mathrm{T}}$ range of $2 \mathrm{GeV} / c, 3 \mathrm{GeV} / c$ and $5 \mathrm{GeV} / c$, from STAR [2], PHENIX [20], and ALICE [19,36] data. The open and solid markers correspond to peripheral and central collisions, respectively 

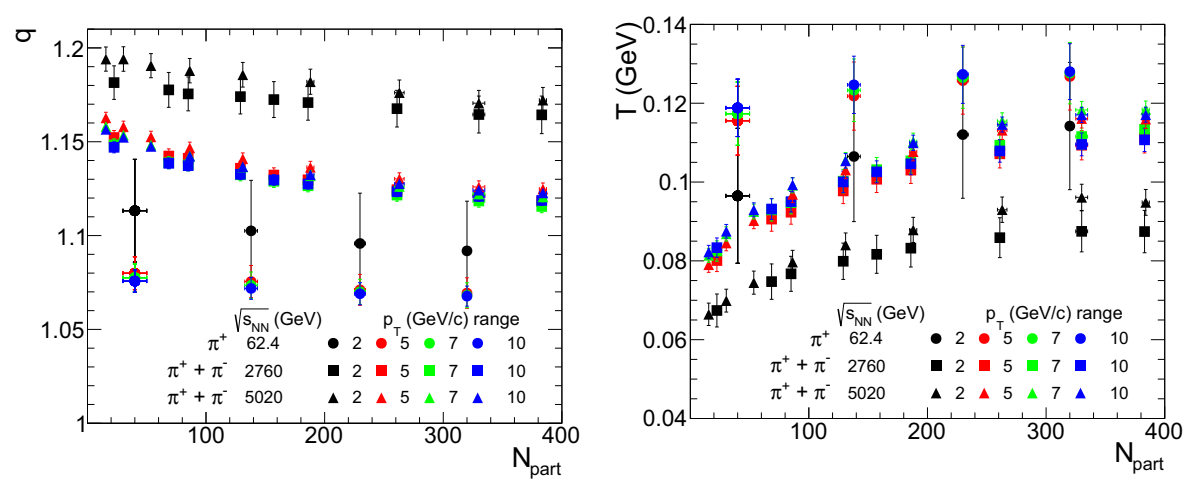

Fig. 8 Tsallis parameters $q, T$ as a function of centrality ( $N_{\text {part }}$ ) for the charged pions at different $p_{\mathrm{T}}$ ranges at $\sqrt{s_{N N}}=62.4,2760,5020 \mathrm{GeV}[19,34,36]$. The different markers and colors represent different $\sqrt{s_{N N}}$ and $p_{\mathrm{T}}$ ranges, respectively

- the fit parameters, $q, T$ depend on the fitting range in $p_{\mathrm{T}}$ for all centralities and collision energies. For the low- $p_{\mathrm{T}}$ region, $q$ values have a big rise and $T$ values have a drop from RHIC to LHC energies. At mid- $p_{\mathrm{T}}$ region $(5-10 \mathrm{GeV} / c), q$ and $T$ are similar (within the uncertainties) at all energies.

- for all centralities, $q$ values are different at low- $p_{\mathrm{T}}$ region $(\sim 2 \mathrm{GeV} / c)$, whereas, at mid$p_{\mathrm{T}}$ region $(5-10 \mathrm{GeV} / c)$ those are same within the uncertainties with higher values. In most of the cases, $q$ decreases from peripheral to central collisions for all $p_{\mathrm{T}}$ ranges.

- the temperature, $T$, is almost constant at mid- $p_{\mathrm{T}}$ region $(5-10 \mathrm{GeV} / c)$ with a higher value than low- $p_{\mathrm{T}}$ fitting range $(\sim 2 \mathrm{GeV} / c)$ for all collision energies. $T$ increases from peripheral to central collisions for all $p_{\mathrm{T}}$ ranges.

The $p_{\mathrm{T}}$ range dependencies of the Tsallis parameters of $\mathrm{Au}-\mathrm{Au}$ collisions at $200 \mathrm{GeV}$ data have similar behavior as that of the Au-Au collisions at $62.4 \mathrm{GeV}$. Since the uncertainty on these datasets are large, only the $\mathrm{Au}-\mathrm{Au}$ data at $62.4 \mathrm{GeV}$ are plotted.

The behaviors of the fit parameters have been explored further by plotting these as a function of the $p_{\mathrm{T}}$ ranges for different collision energies. Depending on the availability of the experimental data, the $p_{\mathrm{T}}$ ranges have been selected up to $2,3,5,7,10,12,15,20$, and $50 \mathrm{GeV} / c$. The evolution of the Tsallis parameters with $p_{\mathrm{T}}$ range selections are shown in Fig. 9. The results shown in the figure correspond to the fit values of all possible $p_{\mathrm{T}}$ spectra at $\sqrt{s_{N N}}=62.4,200,2760$ and $5020 \mathrm{GeV}$ of all charged particles, $\pi^{+}+\pi^{-}$, and $\pi^{+}$. The open and solid markers correspond to peripheral (70-80\%) and most central $(0-10 \%)$ collisions respectively. We observe that

- for peripheral collisions, $q$ initially decreases up to mid- $p_{\mathrm{T}}$ region and then remains constant. For central collisions, $q$ decreases with an increase in $p_{\mathrm{T}}$ up to mid- $p_{\mathrm{T}}$ region, $5-10 \mathrm{GeV} / c$, after which a slow rise is observed.

- for the peripheral collisions, $T$ increases slowly with $p_{\mathrm{T}}$ and then remains constant. For central collisions, $T$ increases with $p_{\mathrm{T}}$ up to the mid- $p_{\mathrm{T}}$ region, and then a slow decreasing trend is observed.

The principal observation from Fig. 9 is that depending on the collision energy and centrality, the $p_{\mathrm{T}}$ spectra have different behavior at different $p_{\mathrm{T}}$ ranges. This is evident from the fact that the particle production mechanisms are different depending on the $p_{\mathrm{T}}$ range probed. 

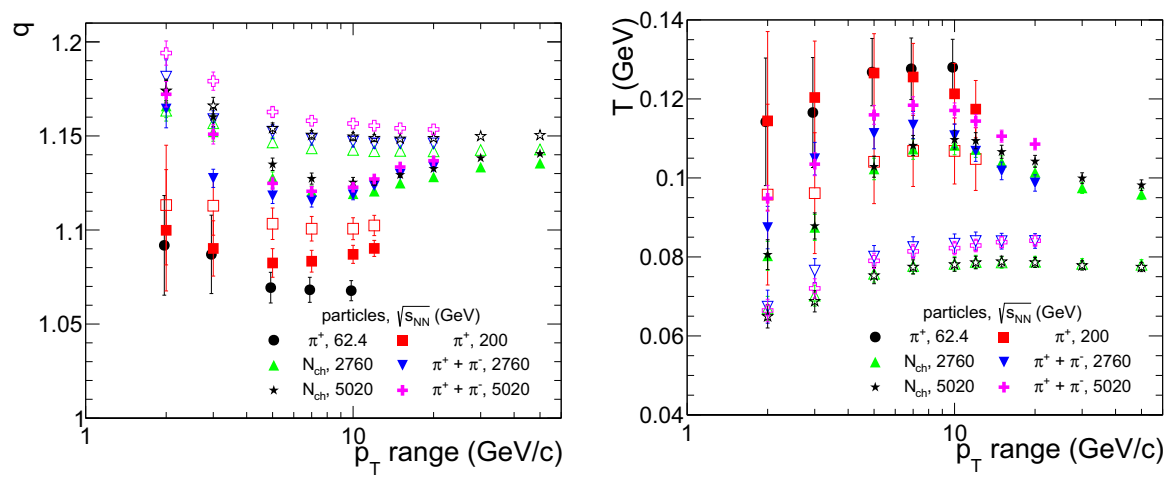

Fig. 9 Tsallis parameters $q, T$ as a function of $p_{\mathrm{T}}$ range for different collision energies. The parameters are at $\sqrt{s_{N N}}=62.4,200,2760,5020 \mathrm{GeV}$ for all charged particles $N_{\mathrm{ch}}$, all charged pions $\left(\pi^{+}+\pi^{-}\right)$and $\pi^{+}$ as labeled by different markers [19,34-38]. The open and solid markers correspond to the parameters of the peripheral and central collisions, respectively

The charged pions observed at lower $p_{\mathrm{T}}$ range are affected by resonance production, flow, coalescence, whereas at mid- $p_{\mathrm{T}}$ region, contributions from hard $\mathrm{pQCD}$ processes and jets start to dominate.

\section{Discussion}

Our study shows that the Tsallis parameters obtained by fitting the $p_{\mathrm{T}}$ distributions vary with respect to the fitting range in $p_{\mathrm{T}}$, centrality of the collision and collision energy. The study of Tsallis parameters with variation of centrality and collision energy as shown in Figs. 5, 6 show that there may have some correlation between the two parameters. This correlation between the Tsallis fit parameters can be better understood by making profile plots of $T$ and $q$, as shown in Fig. 10. In the profile plots, the ellipses corresponding to the one- $\sigma$ uncertainties of the parameters $q$ and $T$ are shown. In the upper panel of Fig 10,T vs. $q$ are plotted for fits up to $p_{\mathrm{T}}$ of $2 \mathrm{GeV} / c$ for pions for all collision energies and collision centralities. We observe that for a given $\sqrt{s_{N N}}$, central collisions have higher effective temperatures compared to those of the peripheral collisions. In addition, with increasing collision energies, $T$ decreases, whereas $q$ increases as has been found before. In the lower panel of Fig 10, we plot the variation of $T$ and $q$ for fit ranges up to $p_{\mathrm{T}}$ of $10 \mathrm{GeV} / c$ for collision energies of $62.4 \mathrm{GeV}$ and above. At the LHC energies, fit values from charged particles and identified charged pions are plotted. The observed pattern is in general, similar to what has been seen for fits with $p_{\mathrm{T}}$ up to $2 \mathrm{GeV} / c$, although the values of $q$ and $T$ are different for the same collision energies. The uncertainties ellipses of the profile plots show that the parameters, $q$ and $T$, values have a mutual dependency and they have anti-correlation. From the lower panel of Fig 10, we observe that for the LHC energies the uncertainties ellipses are very small. Therefore, the Tsallis parameters are independent with precise values.

We have mentioned before that Boltzmann-Gibbs distribution with blast-wave formula has been popularly followed to obtain the temperature at kinetic freeze-out conditions in heavy-ion collisions [2,17-19]. However, it is limited to describe only small $p_{\mathrm{T}}$ ranges. From our study, it is observed that Tsallis distribution fits the spectra of long $p_{\mathrm{T}}$ ranges. The 

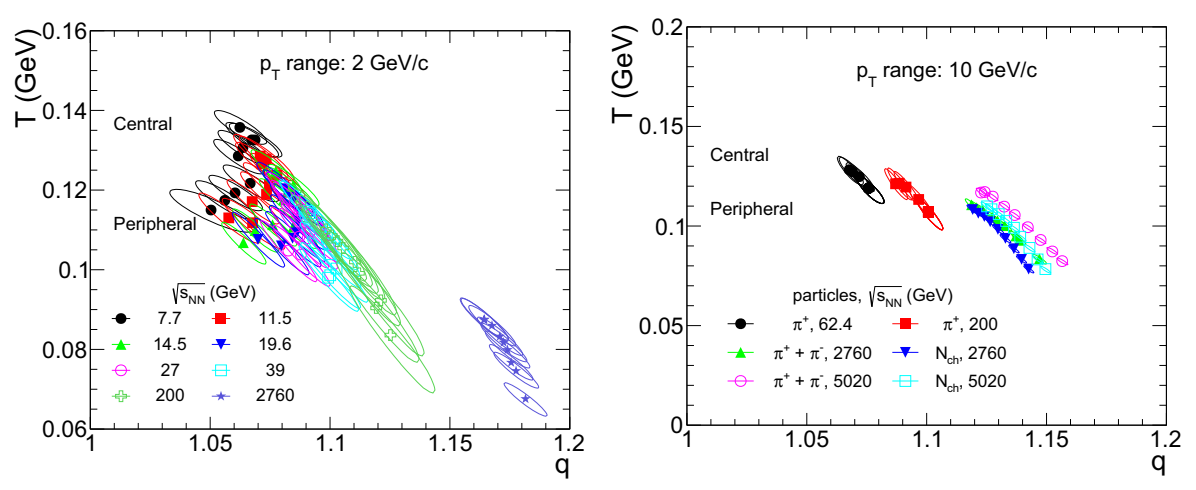

Fig. 10 Profile plots of the temperature $(T)$ and $q$ with one- $\sigma$ uncertainties ellipses for different centrality classes and collision energies for (upper panel) low $p_{\mathrm{T}}$ range (up to $2 \mathrm{GeV} / c$ ) and (lower panel) $p_{\mathrm{T}}$ range up to $10 \mathrm{GeV} / \mathrm{c}$

study of the $p_{\mathrm{T}}$ spectra of the identified particles in the Tsallis framework shows that the effective temperature follows a mass ordering with higher effective temperatures for particles with larger masses as observed in other study [20]. That can be seen from the flattening of the spectra with higher mass particles. It is also observed that spectra of peripheral collisions have better fitting compare to central collisions. This is understood as the effective temperatures from the Tsallis fit include the effect of radial flow. These observations infer that accounting for the radial flow part within the Tsallis distribution functions may better describe the spectra for particles with higher masses and central collisions where radial flow has significant contributions. The inclusion of radial flow may describe the unusual values of $q$ and $T$ for the proton spectra at the LHC energies.

\section{Summary}

In high-energy collisions, the transverse momentum spectra of produced particles provide useful information regarding the particle production mechanisms as well as freeze-out conditions of the system. We have analyzed the $p_{\mathrm{T}}$ spectra of identified and all charged particles produced in $\mathrm{Au}-\mathrm{Au}$ collisions at eight energies at $\mathrm{RHIC}$ and $\mathrm{Pb}-\mathrm{Pb}$ collisions at two energies at LHC using a the non-extensive Tsallis statistics. The Tsallis function is found to be successful to fit a wide range in $p_{\mathrm{T}}$, with fit parameters, $q$, which is the entropy index measuring the degree of non-additivity of the entropy of the system, effective temperature, $T$, and a normalization parameter $V$ which is proportional to the volume of the system.

For each collision centrality, with the increasing of the collision energy, $q$ systematically increases and $T$ decreases. In the centrality dependency study, we found that $q$ is almost constant with an increasing trend for peripheral collisions in LHC energy. The values of $T$ increase from peripheral to central collisions. The parameter, $V$, increases monotonically with centrality and collision energy. Dependence of the parameters, $q, T$, on the fitting range in $p_{\mathrm{T}}$ has been observed, which also depends on the collision energy. For central collisions, the fit parameters are found to have a strong dependence on the fitting ranges of $p_{\mathrm{T}}$; however, for peripheral collisions there is a minor dependency on $p_{\mathrm{T}}$. At mid- $p_{\mathrm{T}}$ range, the Tsallis parameters are found to be constant within the uncertainty. At low $p_{\mathrm{T}}$ ranges, the particle 
production is dominated by soft processes, whereas at mid- $p_{\mathrm{T}}$ ranges, $\mathrm{pQCD}$ processes will have a larger effect. So, this observation may be interpreted as the effect of different physics processes dominating different $p_{\mathrm{T}}$ domains, which also depend on centrality. The profile plot of $T v s . q$ with the uncertainties ellipses shows an anti-correlation of the Tsallis parameters.

In literature, fits of $p_{\mathrm{T}}$ distributions with Tsallis function are found to be successful in describing identified and all charged particles in $p p$ collision system. In the present study, we have found that in heavy-ion collision systems, the Tsallis distribution gives good fits for $p_{\mathrm{T}}$ spectra of pions for full transverse momentum ranges, but the fits are not as satisfactory for kaons and protons, particularly in central collisions. We have also found that the Tsallis distribution provides good fits for full $p_{\mathrm{T}}$ spectra in case of all charged particles. This result is obvious as the pions are the most abundant particles in all charged particles spectra. The centrality-dependent mass ordering in the effective temperature has been observed, which suggests that the contribution of radial flow might need to be incorporated into the standard Tsallis distribution function. Further investigation is needed to include radial flow contributions in order to understand the full nature of the evolving system and freeze-out in relativistic heavy-ion collisions.

Funding Open Access funding provided by CERN.

Open Access This article is licensed under a Creative Commons Attribution 4.0 International License, which permits use, sharing, adaptation, distribution and reproduction in any medium or format, as long as you give appropriate credit to the original author(s) and the source, provide a link to the Creative Commons licence, and indicate if changes were made. The images or other third party material in this article are included in the article's Creative Commons licence, unless indicated otherwise in a credit line to the material. If material is not included in the article's Creative Commons licence and your intended use is not permitted by statutory regulation or exceeds the permitted use, you will need to obtain permission directly from the copyright holder. To view a copy of this licence, visit http://creativecommons.org/licenses/by/4.0/.

\section{References}

1. B. Abelev et al., STAR Collaboration. Phys. Rev. C 79, 034909 (2009)

2. L. Adamczyk et al., STAR Collaboration. Phys. Rev. C 96, 044904 (2017)

3. Y.-Q. Gao et al., Pramana. J. Phys. 79, 1407 (2012)

4. C.Y. Wong et al., Phys. Rev. D 91, 114027 (2015)

5. M.D. Azmi, J. Cleymans, Eur. Phys. J. C 75, 430 (2015)

6. T. Bhattacharyya et al., J. Phys. G: Nucl. Part. Phys. 45, 055001 (2018)

7. M.D. Azmi et al., J. Phys. G: Nucl. Part. Phys. 47, 045001 (2020)

8. J. Cleymans, D. Worku, Eur. Phys. J. A 48, 160 (2012)

9. G. Arnison et al., UA1 Collaboration. Phys. Lett. B 118, 167 (1982)

10. R. Hagedorn, Riv. Nuovo Cim.6N10, 1 (1983)

11. B. Abelev et al., Eur. Phys. J. C 73, 2662 (2013)

12. C. Tsallis, J. Stat. Phys. 52, 479 (1988)

13. C. Tsallis et al., Physica A 261, 534 (1998)

14. H. Zheng and Lilin Zhu, Advances in High Energy Phys. 2016, 9632126 (2016)

15. R. Rath et al., J. Phys. G: Nucl. Part. Phys. 47, 055111 (2020)

16. E. Schnedermann, J. Sollfrank, U.W. Heinz, Phys. Rev. C 48, 2462 (1993)

17. J. Adam et al., STAR Collaboration. Phys. Rev. C 101, 024905 (2020)

18. B. Abelev et al., ALICE Collaboration. Phys. Rev. C 88, 044910 (2013)

19. S. Acharya et al., ALICE Collaboration. Phys. Rev. C 101, 044907 (2020)

20. S.S. Adler et al., PHENIX Collaboration. Phys. Rev. C 69, 034909 (2004)

21. H. Wei et al., Eur. Phys. J. A 52, 102 (2016)

22. H. Lao et al., Nucl. Sci. Tech. 29, 82 (2018)

23. J. Cleymans and D. Worku, J Phys. G: Nucl. Part. Phys. 39, 025006 (2012)

24. B.I. Abelev et al., STAR Collaboration. Phys. Rev. C 75, 064901 (2007) 
25. A. Adare et al., PHENIX Collaboration. Phys. Rev. C 83, 052004 (2010)

26. A. Adare et al., PHENIX Collaboration. Phys. Rev. C 83, 064903 (2011)

27. G. Aad et al., ATLAS Collaboration. New J. Phys. 13, 053033 (2011)

28. A.M. Sirunyan et al., CMS Collaboration. Phys. Rev. D 96, 112003 (2017)

29. K. Aamodt et al., ALICE Collaboration. Eur. Phys. J. C 71, 1655 (2011)

30. B. Abelev et al., ALICE Collaboration. Phys. Lett. B 717, 162 (2012)

31. S. Acharya et al., ALICE Collaboration. Eur. Phys. J. C 78, 263 (2018)

32. S. Acharya et al., ALICE Collaboration. Eur. Phys. J. C 80, 167 (2020)

33. D. Mishra Ph.D. thesis: "Particle Production Studies in Au+Au and U+U Collisions Using the Detector at RHIC and Understanding the Freeze-out Dynamics"

34. B. Abelev et al., STAR Collaboration. Phys. Lett. B 655, 104 (2007)

35. B. Abelev et al., STAR Collaboration. Phys. Rev. Lett. 97, 152301 (2006)

36. J. Adam et al., ALICE Collaboration. Phys. Rev. C 93, 034913 (2016)

37. B. Abelev et al., ALICE Collaboration. Phys. Lett. B 720, 52 (2013)

38. S. Acharya et al., ALICE Collaboration. J. High Energy Phys. 11, 013 (2018)

39. Aleksas Mazeliauskas, Vytautas Vislavicius, Phys. Rev. C 101, 014910 (2020) 